\title{
Digital Image Tamperin Gdetection using sift Key-Point
}

\author{
Anjali Diwan, Rajat Sharma, Anil K Roy, Suman K Mitra
}

\begin{abstract}
Copy-move imitation is a widespread and generally utilized operation to corrupt digital image. It is considered as the most effective research areas in the domain of blind digital image forensics area. Keypoint based totally identification techniques have been regarded to be very environment-friendly in exposing copy-move proof because of their steadiness against a number of attacks, as like large-scale geometric movements. Conversely, these techniques don't have the capabilities to cope with the instances if copy-move forgeries only engage in minor or clean areas, the place the quantity of keypoints is more restricted. To affirm the originality of image, detection of digital image tempering is required. To manage this difficulty, a quick and efficient copy-move imitation detection process is promoted by using the skill of hierarchical function point matching. It is viable to produce an adequate quantity of key points that are present in small or easy areas with the aid of reducing the brightness threshold and resizing the enter digital image. After that, construct a novel hierarchical equivalent technique to remedy the key point equivalent issues over a huge quantity of the key points. To decrease the false alarm charge and exactly localize the affected areas, we similarly advise an innovative iterative localization approach by way of using the steady elements (which comprises of the overriding orientation and the scale data) and the color data of all key point. The proposed technique validates the highest quality overall functioning of the suggested approach in terms of efficiency and precision.
\end{abstract}

Index Terms: Copy-Move, Forgery Detection, Freak Descriptor, SIFT (SIFT Detector), Tampering

\section{INTRODUCTION}

The most regular tamper method is Copy-move imitation in which one can copy one or two sections of a digitalimage and then paste it in any other location of the identical image. The largest trending research domain in blind image forensics is copy-move forgery detection (CMFD). A forgery region might be distinguished if a grouping area delivered by

Revised Manuscript Received on February 15, 2020.

* Correspondence Author

Anjali Diwan*, PhD Scholor, Dhirubhai Ambani institute of Information and communication technology, Email Id - anjali.diwan@ieee.org

Rajat Sharma, M. Tech Student, Dhirubhai Ambani institute of Information and communication technology, Email Id - rajatsharma160996@gmail.com

Anil K Roy, Associate professor, Dhirubhai Ambani institute of Information and communication technology, Email Id - Anil_Roy@daiict.ac.in

Suman K Mitra, Professor, Dhirubhai Ambani institute of Information and communication technology, Email Id - Suman_mitra@daiict.ac.in

(C) The Authors. Published by Blue Eyes Intelligence Engineering and Sciences Publication (BEIESP). This is an open access article under the CC BY-NC-ND license (http://creativecommons.org/licenses/by-nc-nd/4.0/) coordinating sets with a similar relative change is quite high[1].Between the most regularly occurring categories of data frauds are those depends on copy-move, in which the person copies a location of interest in one phase of the image and transmits it to different, using geometric transformations or by executing photograph processing methods i.e., enhancing lighting features and texture, with the aim of covering up more or enjoyable confirmation of the original photograph [2]. The main aim of this domain is to upgrade recognizable proof framework to compute the credibility of pictures and to release the possibility of photograph prevalence imitated. In recent years, so many counterfeit recognization techniques have been developed. The copy-move is described by using replication of the surrounding area of a digital image and inserting to a different area in the identical digital image, generally to conceal unnecessary areas of an image. On the different hand, photograph splicing is the technique of copying an area of an image and pasting it to a different area in some other digital image. In this article, [4] we are retrieving the digital image characteristics and examining it to identify the counterfeit image and additionally deciding the category of the imitation whether it is copy-move or splicing. A lively method to utilizes this prior data, hidden into the photo to check its originality. Such data is hidden with the aid of means of digital watermark or digital signatures [13]. Passive techniques are prioritized over the environment-friendly approach as they do not choose any prior data, but the photo itself. As tampering is no longer recognized visually, passive strategies analyze the underlying statistical characteristic of the digital image which is meant to be in reality disturbed by using the manipulation method. The fingerprint is allocated to all keypoint for the unique recognization of keypoints. To become aware of resemblance amongst copy-paste solid areas they fed to a classifier. The candidates with more classifier scores are calculated to be copy-paste forged. The copy-and-move imitation has several phases of photograph processing. The simplification and minimize of mathematical calculation is executed by means of the use of "Scale-Space Extrema Detection". This builds a scaled-down area by the use of the Dyadic Wavelet transform. DoG (Difference of Gaussian) of image is hidden to dimensionally reduce the photograph house [6]. This article suggests to a photo authentication approach to distribute with snapshots tampered using the copy-move method, which makes a solid photo by way of copying a sure location of a photograph and moving it to any other part of the identical one [7]. Good imitation identification should become aware of the duplicated photograph areas without getting disturbed through minor modifications or functions as adding noise, color modification or compression. 
It is very tedious to pick out these types of damaging by using visual tests up. Moreover, put up processing features such as blurring, contrast discount and geometric features like scaling, zooming, Rotating, reflecting, translating and affine transformations enhances the hardness of the identification two tasks.

In particular time, identifying them is extra difficult, especially if the copy-move imitation solely occupies minor or clean areas, or if the cast areas are operated through using few harsh attacks, as like large-scale resizing and more addition of noises. Nowadays, several digital image copy-move imitation identification methods are suggested, which can be roughly considered i.e., dense-field (or block-based) techniques and sparse-field (or keypoint-based) methods.

\section{RELATED WORK}

To execute the function of colours image copy-move counterfeit identification, first, the test image is modified into the comparable YCbCr color model from its original color system. After the execution of the procedure, replicate areas of the image are obtained from the image itself, comparable areas in the same digital image are acquired [12]. It is difficult to compute the working of locales sets examination of different sizes because the size of these copied zones is obscure. Along these lines, to give more viability, in our suggested method, the digital image is sub-isolated into covering squares of steady size. At that point, to extract the highlights from the DCT coefficient, a DCT is executed to these units which are relying upon the Zigzag technique to diminish or pack the data (vitality) of every unit and gather it into lesser coefficients. The quick K-mean is executed as this is the good bunching technique to limit the time cost, i.e., used in bunch the units vectors to adapt $\mathrm{K}$ group of highlights, from that point onward, these vectors are arranged lexicographically. To reduce the fake copy-move counterfeit detection the connection between each pair of comparative same kind of vectors is processed. The Principal Component Analysis (PCA) calculation for the recognizable proof of cloning fabrication is the base of this examined article. The PCA method will denote the reproduction component through black color. The GLCM technique is executed [11] for distinguishing the pieces of proof of textural highlights of the info digital image which portrays the ongoing region for the acknowledgment of cloning imitation. At precise roles, an image is associated with every other, via which the texture aspects are computed interior statistical texture testing. The fundamental approach, Principal component evaluation (PCA) and the gray stage co-occurrence matrix (GLCM) is utilized to take out the feature. The records associated with the locations of pixels whichare having an equivalent gray stage rate are furnished through GLCM. A matrix, which has a comparable amount of rows and columns and the quantity of grey tiers current in a photo is termed as GLCM. In the photograph processing, there is fast development in the technological know-how and also there is development in the cameras because of which giant quantity of image are produced barring any research because of which it enhances the extra requirement for the computerized imitation identification process, so the steadiness of an original photo is decided [14]. In the current research, the detection of imitation used to be achieved manually. Nowadays, a huge amount of statistics is saved in the digital layout due to which safety issues are increasing. The digital imitation methods are analyzed by means of the Digital Image Forensics (DIF) which is recognized as the protection of image. Active and passive methods are the categories of digital photo imitation identification methods [5].The pre-processing of a digital photo is obligatory in the existing technique method as like watermark embedding or signature production, because of which their applications in the real-world are restricted [6].

To locate the recognizable proof and confinement of video generation strikes, we enlarge the method two suggested in [7] for nonetheless snapshots because of its dense-field quality and being proficient with both added substance and occlusive duplicate moves. Hence, our suggested method [10] has the three normal stages which are mentioned below-

a) Characteristics extraction;

b) Equivalent feature;

c) Post-handling of the closest neighbour territory.

To get benefits of the previous knowledge, we cross from the identical fundamental equipment [14]. Moreover, SIFTting from nonetheless image to videos, greater new problems rise, that should be explained particularly. Features should be modified to provide the required steadiness to both temporal and spatial damages; Patch Match itself needs to be tailored to deal correctly with a video source; the post-processing needs to be tailor-made to take care of more quantity of wrong hits growing naturally in a rather surplus source. Calculational efficiency ought to be carried on. At trendy frame rates, 60 seconds of the video relates to about 1500 frames and can quickly turn into uncontrollable even for quick YouTube TM videos. The complete problems are explained in the below-mentioned areas.

In the suggested device [8] present reproduction digital image is modified into a dark scale digital image at that point minute's standardized infer and famous difference are taken out as capacity vector for image in the information gathering. The relation between the imply and trendy divergence is considered. Grouping is a function of growing a set of bodily or summary factors into two types of comparable elements. A group is a collection of factors having the same factors. BIRCH process will exhibit the group function as a hierarchy. It receives records spots, linear sum and addition of data factors as input. The output value is same image existing as group characteristic hierarchy with mother or father-child connection amongst forged image and unique image. The group is a built-in model of equal statistics elements. A group of data factors is considered together as a single team and so may additionally be measured as a type of fact compression. The BRICH grouping process is the easiest unsupervised grouping process. It receives the enter factor and linear sum with a team of $\mathrm{n}$ elements into a total of true spots. It is accomplished on fused groups such that the producing intergroup resemblance is more except the intergroup resemblance is less. Initially, the process arbitrarily chooses input snapshots referred with the aid of corresponding regular mean, each of which in the beginning denotes a group means or center. After that the subsequent group of the balanced digital image is realized and allocated to the group to which resembles it closely, relying on the distance amongst the aspect as well as the group mean. It calculates the new suggestions for every set. This method proceeds until the standard work meets. 


\section{METHODOLOGY}

The proposed method is based on an effective and precise keypoints technique for image copy-move counterfeit recognization and localization., accomplishing reliably progressively better execution regardless of whether the duplicate move imitation just as smooth or little territories or the produced image have been handled by scarcely any troublesome assaults (e.g., huge scale resizing and overwhelming commotion expansion). Fig. 1 shows the system of our proposed digital image imitation distinguishing proof strategy, which pursues the great work process, in particular,

1. Define detector (SIFT detector)and descriptor (FREAK)

2. Read ground truth and define factor for clustering

3. Detect key-point from the image using Kp detector

4. Use the FREAK descriptor and for all the key points to find the k-NN feature.

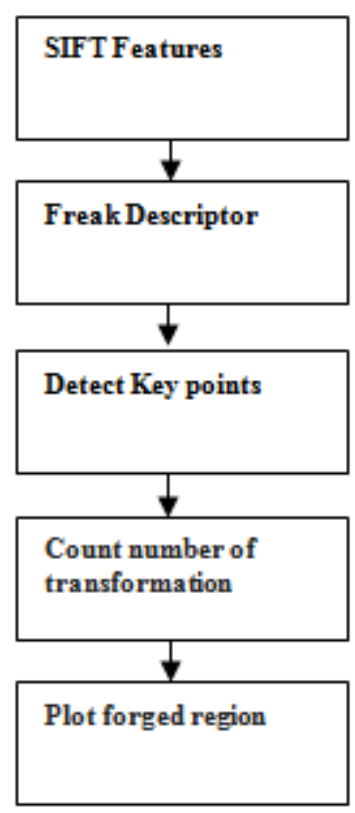

\section{Figure.1 Algorithm of the proposed system}

5. Discard the nearest key-point up to threshold so that it will not take similar points near it.

6. Operate agglomerative clustering

7. Count the number of transformations on the image that has completed.

8. Plot forged region with points based on clustering any clustering and segmentation procedures.

By completely utilizing the strength qualities (counting the scale data and key direction) and the shading data of every keypoint, our proposed strategy achieves great exactness of location results at an extensively decreased computational expense.

\section{A.SIFT}

First approach in our method is based on scale and rotation invariant SIFT feature. These features are illumination invariant too. We detect stable local keypoints and a feature vector is computed from a locak pixel area around that keypoint. Constructing a scale space: The initial preparation is done by generating a scale space.

This ensures scale invariance in internal representations of the original image. Let's consider an input image $\mathrm{I}(\mathrm{x}, \mathrm{y})$ and

scale space of the image I is defined as convolution of I with $\mathrm{G}$ (Gaussian function) with scale factor $\sigma$ :

$$
S(x, y, \sigma)=G(x, y, \sigma) * I(x, y)
$$

The Laplacian of Gaussian is great for finding interesting points (or key points) in an image. But it's computationally expensive. So to calculate it quickly difference between consecutive scale is calculated and is called Difference of Gaussian. DoG of images are approximately equivalent to LoG with less computational complexity. Gaussian function $\mathrm{G}$ is defined as :

$$
G(x, y, \sigma)=\frac{1}{2 \pi \sigma^{2}} e \frac{-\left(x^{2}+2\right)}{\sigma^{2}}
$$

If Scale factor $\sigma$ in denominator is removed we can get scale in variant Laplacian of Gaussian.

Finding keypoints: Kepoints are maxima and minima in the Difference of Gaussian image we calculate in two steps firest calculate maxima and minima by iterating through each pixel and it's neighbour. This is done for current image and image scale above and below it. After that using available data sub pixel values are generated around approximate keypoint.

Edges and low contrast regions are bad keypoints. Eliminating these makes the algorithm efficient and robust. An orientation is calculated for each key point based on the local image gradient direction magnitude. A gradient orientation histogram in the neighborhood of the keypoint is calculated. For image $S(x, y, \sigma)$ at keypoint scale $\sigma$ gradient magnitude and scale is calculated.

Table 1 All Images of the dataset

\begin{tabular}{|l|l|l|}
\hline Dataset & Proposed Approach & $\begin{array}{l}\text { Time per } \\
\text { image }\end{array}$ \\
\hline F600 & 92.71 & $1350 \mathrm{~s}$ \\
\hline CMFD & 91.11 & $1224 \mathrm{~s}$ \\
\hline Coverage & 86.18 & $1351 \mathrm{~s}$ \\
\hline F220 & 80.64 & $1324 \mathrm{~s}$ \\
\hline
\end{tabular}

Also, typical photograph image are slanted to be most lightened in the inside and dimmer in the outline. Three datasets namely F600, CMFD, and F220 are utilized. The values of the SIFT-freak descriptors are tabulated in table 1.

\section{B.FREAK DESCRIPTOR}

Fast Retina Keypoint is shortly called as FREAK descriptor. The FREAK descriptor is utilized to find a k-NN feature. Figure 2 shows the freak descriptor.

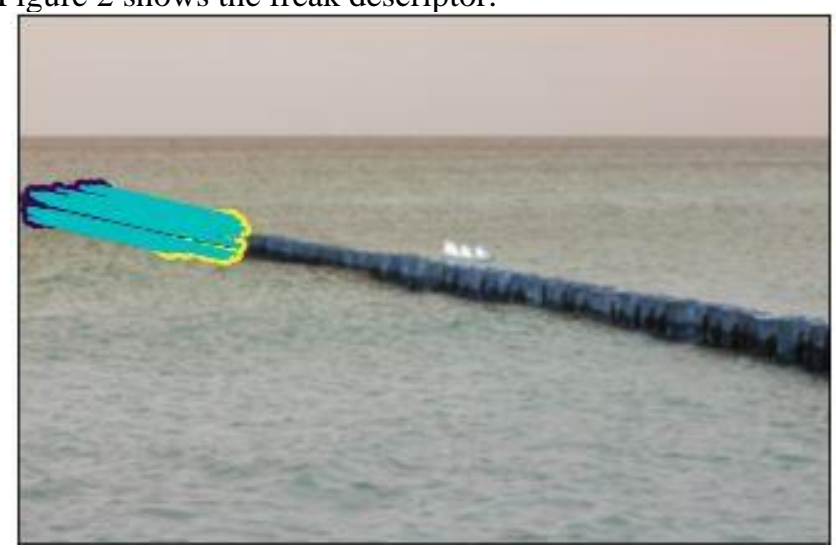

Figure 2: Beach wood

Published By: 
The proposed method recommends a keypoint descriptor animated by the human-visual framework and the most accurate retina, authored Fast Retina Key-point (FREAK). By adequately looking at digital image forces over a retinal testing structure a cascade of binary strings is calculated.

FREAKs are normally snappier to figure with decreased memory load and furthermore more solid than Scale-invariant Feature Transform, Speeded Up Robust Features or Binary Robust Invariant Scalable Keypoints. They are focused choices to introduce keypoints in explicit for implanted applications. These descriptors are applied to the dataset and few examples are shown in fig 2 and fig 3. The rotation value and the scale of rotation are tabulated in table 2 .

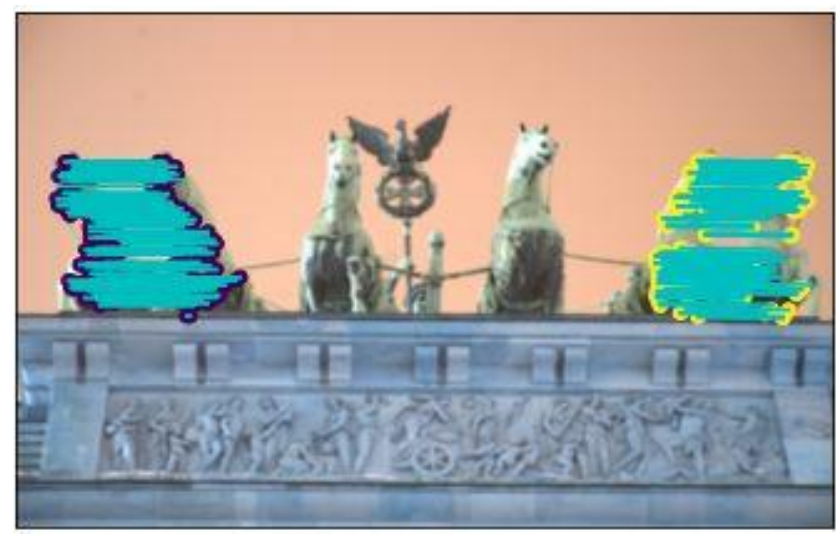

Figure 3 Horse Copy

Table 2: The rotation value and the scale of rotation are tabulated

\begin{tabular}{|l|l|}
\hline RotationAngle & Proposed Approach \\
\hline $\mathbf{2}$ & 87.05 \\
\hline $\mathbf{4}$ & 87.05 \\
\hline $\mathbf{6}$ & 87.05 \\
\hline $\mathbf{8}$ & 87.05 \\
\hline $\mathbf{1 0}$ & 87.05 \\
\hline $\mathbf{2 0}$ & 85.71 \\
\hline $\mathbf{6 0}$ & 84.77 \\
\hline $\mathbf{1 8 0}$ & 84.77 \\
\hline
\end{tabular}

The rotation and its scale values of the F600 dataset alone are found and tabulated in table no. 3

Table 3: F600 dataset rotation and scale values

\begin{tabular}{|l|l|}
\hline F600 dataset & Proposed \\
\hline Rotation & 90.16 \\
\hline Rotation and scale & 87.67 \\
\hline
\end{tabular}

Characteristics are the data taken out from image in terms of numerical value. Typical image features are an edge, corner, blob, etc. Generally, Features are also termed as descriptors. Two methods are available to represent the features of the image i.e., Global feature and local feature.

1. Global component: Global Feature portrays the digital image all in all to sum up the whole item. Global Feature incorporate form portrayals, shape descriptors, and surface characteristics. Few instances of Global descriptors are HOG, Invariant Moments (Hu, Zerinke), Co-HOG, and Shape Matrices.
2. Local feature: Digital image Patches are depicted by Local feature. A few examples of local descriptors are FREAK, SURF, BRIEF,BRISK, SIFT, and SIFT.

3. The radius of the centerr ${ }_{C}$ will be taken as the size of each cell in the original FREAK [17]. The standard deviation of the corresponding Gaussian can be approximated as follows Equation (2) :

$$
\sigma_{c}=\frac{r_{c}}{3}
$$

4. The relative surround extent has a wide range across retinal ganglion cells. We have empirically chosen the surround to be double size of the center, since the DoG behaves as an edge detector and this is the functionality we are interested in. Therefore, the standard deviation of the surround can be obtained as follows Equation (3):

$$
\frac{\sigma_{s}}{\sigma_{c}}=2 \text { (3) }
$$

5. From this equation, the size of the surround can be obtained replacing Eq. 3.The formula for the Difference of Gaussians (DoG) is the following Equation (4):

$$
K(x, y)=w_{C} G_{\sigma C}(x, y)-w_{S} G_{\sigma S}(x, y)
$$

6. Where, $\mathrm{w}_{\mathrm{C}}$ and $\mathrm{w}_{\mathrm{S}}$ are constants, which determine the type of features estimated by this Filtering stage [18].

\section{C.AGGLOMERATIVEHIERARCHICAL CLUSTERING}

The most far-reaching classification of progressive clustering is an Agglomerative bunching which is used to amass components in groups rely upon their likeness. It's likewise alluded to as AGNES (Agglomerative Nesting).

The calculation SIFTts by considering every component as a singleton group. Succeeding, sets of groups are progressively joined until one major bunch containing all components is generated. The outcome is Dendrogram which is a tree-based portrayal of the components.

Agglomerative clustering works in a "bottom-up" style. i.e., every element is first treated as a single-element cluster (leaf). Following, Nodes is obtained by merging two comparative images to get one large cluster. The upper mentioned process is repeated until Root is obtained which is the sole large cluster comprising all small cluster of the image.

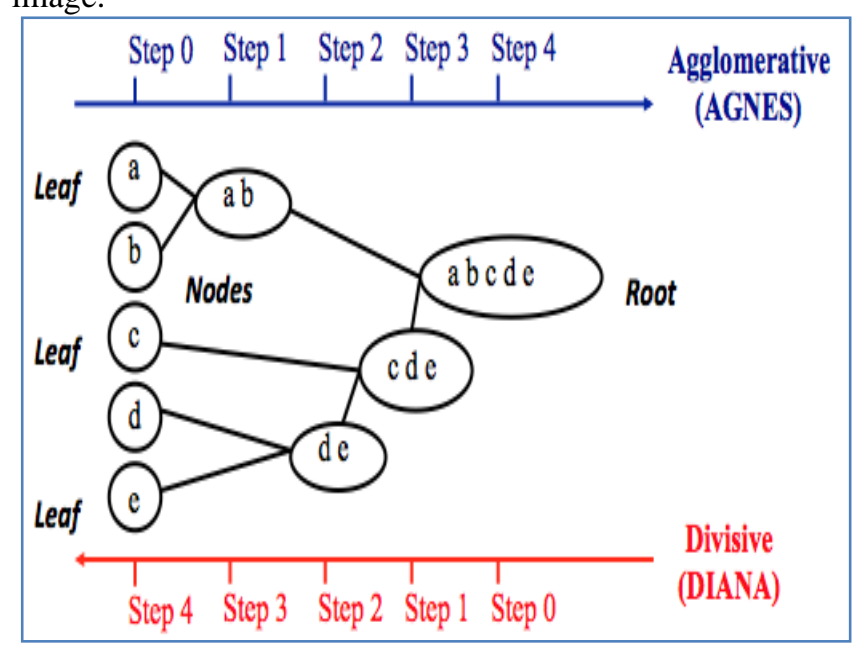

Figure 4: Showing AGNES and DIANA 
Divisive Analysis is also known as DIANA which is the opposite of agglomerative clustering and it works in a "top-down" manner.

As shown in figure 3 it SIFTts from the root, at this stage, the whole element is present in one bunch. At every succeeding stage of division, the most mixed bunch is separated into two smaller bunches. The function is repeated until all components are having their own single bunch (see figure 3 above).

\section{Steps to Agglomerative Hierarchical Clustering:}

1. Setting up the information

2. From the data, group discordance is calculated among each pair of elements

3. At this Stage, linkage function is used to assemble components into various leveled group tree, in view of the separation data created at stage 1. Linkage Function is used for connecting Components/groups that are in nearness.

4. The various leveled tree is divided into groups which makes a segment of the information.

\section{EXPERIMENTAL RESULT}

In this Section, we evaluate the proposed copy-move forgery detection method. The detection performance is measured at both the image level and the pixel level. At the image level, we focus on the ability that an image can be correctly recognized as forged or genuine; at the pixel level, we analyze the performance for accuracy

$$
\begin{gathered}
\text { Accuracy }=\frac{(T P+T N)}{(T P+F P+F N+T N)} \\
\text { True positive Rate }=\frac{T P}{(T P+F N)} \\
\text { False Positive Rate }=\frac{F P}{(F P+T N)} \\
\text { Sensitivity }=\frac{T P}{(T P+F N)} \\
\text { Specificity }=\frac{T N}{(F P+T N)}
\end{gathered}
$$

F1 score, which gives the synthetical performance through a single value. Mathematically,

$$
\begin{aligned}
\text { Recall } & =\frac{T P}{T P+F N} \\
F & =2 \cdot \frac{\text { precision } \cdot \text { recall }}{\text { precision }+ \text { recall }}
\end{aligned}
$$

Precision $=\frac{T P}{T P+F P}$

This proposed method is used to measure the Accuracy, Sensitivity, Specificity, Precision, Recall and F1 Score as shown in Table 4 and Figure 5. Compare the Existing methods and proposed Methods as shown in Table 5 and Figure 6.

Table 4 Average Results of Proposed Method in Percentage

\begin{tabular}{|l|l|}
\hline Measure & Proposed Method \\
\hline Accuracy & 96.15 \\
\hline Sensitivity & 97.64 \\
\hline Specificity & 93.47 \\
\hline Precision & 96.51 \\
\hline Recall & 97.64 \\
\hline F1 score & 97.07 \\
\hline
\end{tabular}

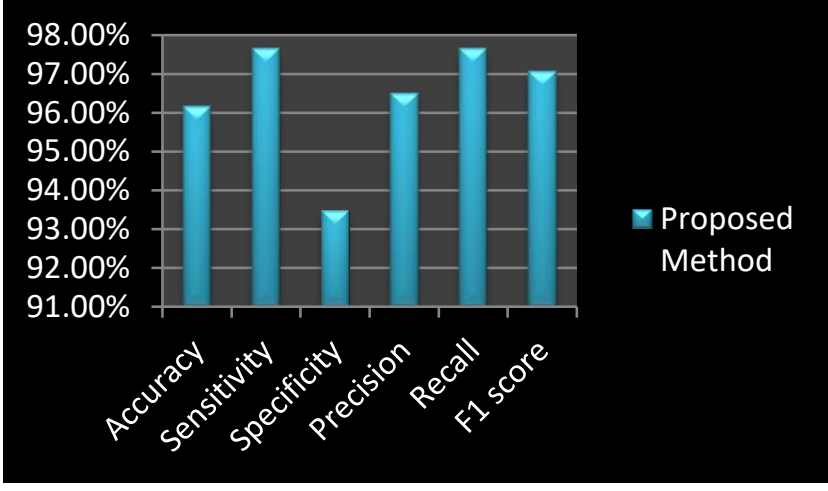

Figure 5 Proposed Method

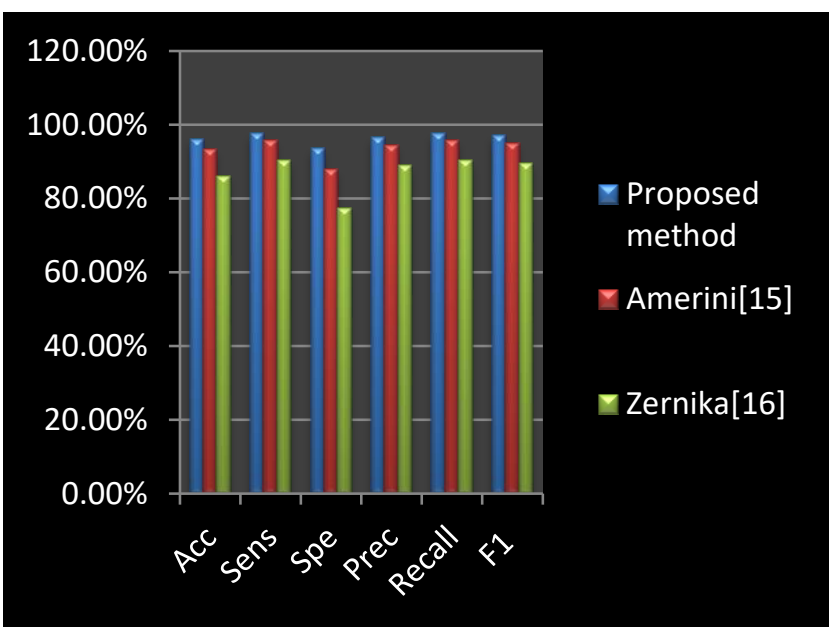

Figure 6 Comparisons of result with recent published approaches

\section{CONCLUSION}

This article creates awareness about a quick and efficient copy-move imitation identification method. By cautiously analyzing the freak descriptors, we have first described that it is viable to produce an adequate volume of keypoints in easy or minor areas, by decreasing the contrast threshold and altering the size of the digital image. Scale-Invariant Feature Transform (SIFT) Features are extracted. Then a novel Agglomerative Hierarchical Grouping technique has been advised to improve the integral alike issues. To diminish the bogus alert expense and definitely center the replicated zones, we have suggested a novel iterative restriction approach without considering any grouping and division strategies. By using the steadiness houses of the SIFT process completely (comprising the major working and the scale data) and the shade records of every keypoint, the suggested method obtains extra appropriate identification precision. The process is performed to three exceptional datasets namely CMFD DATASET, GRIP DATASET, and MICCF220 DATASET. Broad trial results have been done to depict increased overall performance of the suggested method.

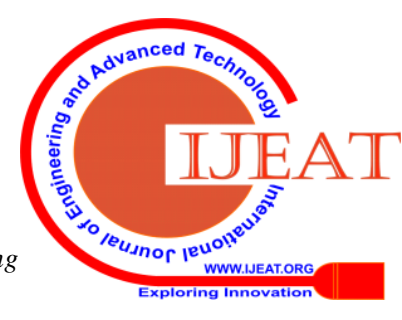




\section{Digital Image Tampering detection using SIFT key-point}

\section{REFERENCES}

1. JunlinOuyang, Yizhi Liu, Miao Liao, School of Computer Science and Engineering, "Copy-Move Imitation Detection Based on Deep Learning", 2017 10th International Congress on Digital image and Signal Processing, BioMedical Engineering and Informatics (CISP-BMEI 2017)

2. Guilherme L. Santos, Giovanni R. Oliveira, Fernando F. Prado, Rafael Serikaku, Ricardo M. Santos "“ A Bio-Inspired Methodology for Digital Imaging Forensic Detection,” WVC 2019

3. RoqayaHamadJaafar ,Zahraa. H. Rasool , Abbas H. HassinAlasadi , "New Copy-Move Imitation Detection Process," 2019 International Russian Automation Conference (RusAutoCon)

4. Youssef William, SherineSafwat, Mohammed A.-M. Salem "Robust Digital image Imitation Detection Using Point Feature Analysis," Proceedings of the Federated Conference on Computer Science and Data Systems.

5. NavdeepKanwal, AkshayGirdhar, LakhwinderKaur, “ Detection of Digital Digital image Imitation using Fast Fourier Transform and Local Features," 2019 International Conference on Automation, Computational and Technology Management (ICACTM) Amity University.

6. JitendraRavan, Dr.Thanuja.: Digital image imitation Detection against Forensic Digital image Digital Tampering, 2018 International Conference on Computational Techniques, Electronics and Mechanical Systems (CTEMS).

7. Kelsey Ramirez-Gutierrez, Mariko-Nakano, Gabriel Sanchez-Perez, Hector Perez-Meana.: Copy-Move Imitation Detection Process using Frequency Transforms, SURF, and MSER, Founding by Conacyt, Mexico.

8. G.Nirmala and K.K.Thyagharajan.: A Modern Approach for Digital image Imitation Detection using BRICH Grouping based on Normalised Mean and Standard Deviation, International Conference on Communication and Signal Processing, April 4-6, 2019, India.

9. Yuanman Li, Jiantao Zhou.: Fast and Effective Digital image Copy-Move Imitation Detection via Hierarchical Feature Poin Matching. IEEE Transactions On Data Forensics And Security

10. Luca D'Amiano, DavideCozzolino, Giovanni Poggi, and Luisa Verdoliva: A PatchMatch-based Dense-field Process for Video Copy-Move Detection and Localization. IEEE Transactions on Circuits and Systems for Video Technology

11. TaranjitKaur, AkshayGirdhar, GeetikaGupta"A Robust Process For The Detection Of Cloning Imitation", 2018 Ieee International conference on computational intelligence and computing research.

12. Mokhles Hussein Khudhur, JumanaWaleed, HiyamHatem "An Efficient and Fast Digital Digital image Copy-Move Forensic Technique, 2nd International Conference for Engineering, Technology, and Science of Al-Kitab University 4 -5 December-2018.

13. K. Sreenivas and V. K. Prasad, "Fragile watermarking schemes for digital image authentication: a survey," International Journal of Machine Learning and Cybernetics, vol. 9, no. 7, pp. 1193-1218, 2018

14. D. Cozzolino, G. Poggi, and L. Verdoliva, Efficient dense-field copy move imitation detection," IEEE Transactions on Data Forensics and Security, vol. 10, no. 11, pp. 2284-2297, November 2015.

15. Amerini, L. Ballan, R. Caldelli, A. Del Bimbo, and G. Serra, "A sift-basedforensic method for copy-move attack detection and transformation recovery," IEEE Trans. Inf. Forensics Security, vol. 6, no. 3, pp. 1099-1110, Sep. 2011.

16. J. Li, X. Li, B. Yang, and X. Sun, "Segmentation-based image copymove forgery detection scheme," IEEE Trans. Inf. Forensics Security, vol. 10, no. 3, pp. 507-518, Mar. 2015.

17. S.Khachikian, M.Emadi, "Applying FAST \& FREAK Algorithms in Selected Object Tracking", International Journal of Advanced Research in Electrical, Electronics and Instrumentation Engineering , Vol. 5, Issue 7, July 2016, DOI:10.15662/IJAREEIE.2016.0507003 5829.

18. Cristina Hilario Gomez, KartheekMedathati, " Improving FREAK Descriptor for Image Classfication", 2015, DOI: 10.1007/978-3-319-20904-3.

19. MosalamEbrahimi , Walterio W. Mayol-Cuevas , "Speeded Up Surround Extreme Feature Detector and Descriptor for Real time Applications", 2008.

20. MotilalAgrawal, Kurt Konolige, Morten Rufus Blas, "SIFT: Scale-Invariant Feature Transforms for Realtime Feature Detection and Matching", LNCS 5305, pp. 102-115, 2008.

\section{AUTHORS PROFILE}

Anjali Diwan, PhD Scholor, Dhirubhai Ambani institute of Information and communication technology,

She has served in several reputed organizations in different states in India for various administrative, educational teaching and research assignments. Her area of research include Nanotechnology, image and video processing, signal processing, solar cell and embedded system.

Rajat Sharma, M.Tech Student,Dhirubhai Ambani institute of Information and communication technology

Anil K Roy, Associate professor,Dhirubhai Ambani institute of Information and communication technology

Suman K Mitra Professor,Dhirubhai Ambani institute of Information and communication technology
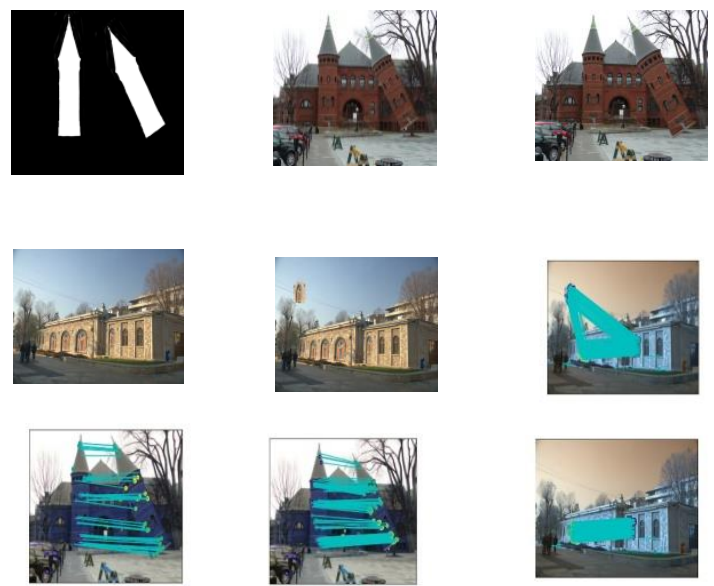